\title{
Persone come noi: Intervista a Mariella
}

Giornale di Tecniche Nefrologiche e Dialitiche 2019, Vol. 3I(2) |40-14|

(C) The Author(s) 2019

Article reuse guidelines:

sagepub.com/journals-permissions

DOI: $10.1177 / 0394936219851983$

journals.sagepub.com/home/gtn

(S)AGE

\section{Le difficoltà nel conciliare terapia enzimatica con la quotidianità quando la terapia domiciliare non è prevista dalla regione di residenza}

\section{Raccontaci, Mariella, in che modo la Malattia di Anderson-Fabry ha coinvolto la vostra famiglia}

Mi chiamo Mariella, ho 49 anni, e ho la malattia di Fabry. Una malattia genetica rara, di cui sono affetti anche i miei tre figli di 15,20 e 21 anni.

Questa patologia non ha cura, ma esiste una terapia che ne limita gli effetti. Nel nostro caso viene trattata con infusioni dell'enzima che il corpo non produce, a cui ci dovremo sottoporre per tutta la durata della nostra vita. Oltre a me, due dei miei figli vengono sottoposti al trattamento, che viene somministrato ogni due settimane impegnando almeno una mezza giornata in ospedale.

\section{Curarsi è dunque un impegno gravoso...}

Abbiamo sostenuto negli anni un lungo pellegrinaggio alla ricerca di una diagnosi, che abbiamo ricevuto solo nel 2014, e di un Centro ad alta specializzazione in grado di seguirci, che tuttora non esiste. Ora siamo sottoposti ad un analogo calvario solo per impedire che la malattia e la gestione della terapia abbia la meglio su di noi.

\section{Come conciliate la vostra vita con la terapia?}

Purtroppo abitiamo in una regione dove la terapia non viene erogata a domicilio. Risiedo a Cervia e la terapia viene erogata nell'Ospedale di Ravenna, a circa 30 chilometri di distanza. Molto spesso le date di somministrazione tra di noi non coincidono e quindi solitamente ci troviamo a dover raggiungere l'ospedale in giorni diversi, con un impiego di tempo che può arrivare fino a nove mezze giornate al mese. Questo comporta non pochi problemi alla nostra famiglia. A causa della perdita di giorni di lavoro, ad esempio, ho dovuto lasciare diverse occupazioni non riuscendo a giustificare le ripetute assenze, dal momento che la patologia non viene riconosciuta per accedere alla
L.104. I miei figli, invece, sono stati costretti a perdere diverse giornate di scuola e a recuperare le lezioni con lo studio individuale o con lezioni di ripetizione assistita. E ora che lavorano stanno vivendo i miei stessi problemi. Per non parlare dei costi, sia per le spese per la trasferta (400$550 \mathrm{~km}$ al mese, per un costo totale di 60-80 Euro) sia per la necessità di avere a disposizione una macchina, cosa per noi non sempre possibile, o un autista. Inoltre, spesso devo ricorrere all'aiuto di un accompagnatore, non potendo guidare a causa delle conseguenze importanti dovute alla malattia, come forti dolori e altri malesseri; la terapia poi mi provoca una forte sonnolenza. Questo complesso di fattori rende spesso molto difficile per me affrontare il viaggio, aumentando i rischi di incidenti stradali lungo il percorso. Così diventa difficile mantenere la necessaria continuità nella terapia.

\section{Ritieni che la somministrazione a domicilio della terapia potrebbe migliorare qualcosa?}

Certo. E' una strada percorsa in moltissime regioni in Italia, che ha il vantaggio di migliorare la qualità della vita di noi pazienti, ma anche di diminuire i costi per la sanità regionale. Penso che, ricevendo la terapia al domicilio, sia io che i miei figli potremmo convivere meglio con la nostra malattia e con la terapia che dovremo effettuare per tutta la nostra vita, evitando le assenze da scuola e dal lavoro, e limitando la 'dipendenza' psicologica dalla malattia.

\section{Secondo te, esistono alternative valide alla terapia domiciliare per la tua famiglia?}

Nello scorso mese di dicembre ho inviato una lettera alle autorità sanitarie della mia regione, che mi hanno risposto proponendomi l'ipotesi di ricevere la terapia in una struttura ospedaliera più vicina a casa. Ma questo non cambierebbe di molto la nostra attuale situazione di 
difficoltà e di disagio, perché la nostra vita rimarrebbe in ogni caso segnata dalla frequentazione dell'ospedale che, oltretutto, fissa le date della somministrazione sulla base della sua organizzazione, senza tenere conto fino in fondo delle nostre esigenze o problematiche, e non migliorerebbe la nostra qualità di vita, già pesantemente segnata dalla malattia. Ricordiamoci che la terapia viene somministrata ogni 14 giorni, che significa 26 infusioni in un anno. Senza la possibilità di effettuare la terapia domiciliare, in un anno ci rechiamo quindi in ospedale per ben 26 giorni, a cui si devono aggiungere ulteriori giornate per visite periodiche di controllo.

Spero che un giorno anche la regione Emilia Romagna renda possibile la terapia domiciliare come avviene in moltissime altre regioni italiane, per permettere alla mia famiglia una migliore qualità di vita.

Grazie Mariella, per la tua disponibilità a raccontarci la tua significativa esperienza, che rispecchia il vissuto di molti pazienti residenti nelle regioni che non permettono di scegliere la terapia domiciliare come alternativa alla terapia in ospedale. Su questo fronte, AIAF proseguirà la sua campagna di sensibilizzazione in collaborazione con le altre associazioni di pazienti lisosomiali, affinchè il diritto di "scegliere" venga esteso in egual modo in tutte le regioni italiane.

Intervista raccolta da Stefania Tobaldini In collaborazione con il Comitato Editoriale 